\title{
Newly identified energy levels and calculated transition rates in astatine atom, the rarest element on Earth
}

\author{
Pascal Quinet ${ }^{\mathrm{a}, \mathrm{b}, *}$, Guillaume Sicorello ${ }^{\mathrm{a}}$ \\ a Physique Atomique et Astrophysique, Université de Mons, Mons, B-7000 Belgium \\ ${ }^{\mathrm{b}}$ IPNAS, Université de Liège, Liège, B-4000 Belgium
}

\section{A R T I C L E I N F O}

\section{Article history:}

Received 8 August 2017

Revised 12 September 2017

Accepted 12 September 2017

Available online 14 September 2017

\begin{abstract}
A B S T R A C T
A theoretical investigation of the atomic structure and radiative parameters involving the lowest states within the $6 p^{5}, 6 p^{4} 7 s$ and $6 p^{4} 7 p$ configurations of neutral astatine is reported for the first time in the present paper. Using different physical models based on the pseudo-relativistic Hartree-Fock approach, the influence of intravalence, core-valence and core-core electron correlation on the atomic parameters is discussed in detail. This work allowed us to establish the intermediate-coupling composition and to clearly fix the spectroscopic designation of six excited levels within the $6 \mathrm{p}^{4} 7 \mathrm{~s}$ and $6 \mathrm{p}^{4} 7 \mathrm{p}$ configurations which had been located in previous experimental studies. In addition new tentative identifications of four levels belonging to the $6 p^{4} 9 p$ and $6 p^{4} 10 p$ configurations are reported in this work together with predicted level energies along the $6 \mathrm{p}^{4} n \mathrm{p}$ and $6 \mathrm{p}^{4} n$ d Rydberg series up to $n=50$.
\end{abstract}

(C) 2017 Elsevier Ltd. All rights reserved.

\section{Introduction}

In view of the high radioactivity of all its isotopes, astatine $(Z=85)$ is the rarest naturally occurring element on the Earth's crust with an estimated total abundance of less than one gram at any given time [1]. This element was discovered in 1940 by Corson et al. [2] by bombarding a bismuth target with $\alpha$ particles. As described by Fry and Thoennessen [3], thirty-nine astatine isotopes with atomic masses ranging from $A=191$ to $A=229$ have been discovered so far and, according to the Hartree-FockBogoliubov (HFB-14) mass model [4], about 37 additional astatine isotopes could exist. All of these isotopes are characterized by very short half-lives, the most stable species being ${ }^{210}$ At with a half-life time of only $8.1 \mathrm{~h}$. This isotope's primary decay mode is positron emission to the relatively long-lived and very toxic ${ }^{210} \mathrm{Po}$. In total, only five isotopes of astatine have half-lives exceeding one hour, namely those between $A=207$ and $A=211$. The least stable ground state isotope is ${ }^{213} \mathrm{At}$, with a half-life of about $125 \mathrm{~ns}$. It undergoes $\alpha$ decay to the extremely long-lived isotope ${ }^{209} \mathrm{Bi}$. With a half-life of $7.2 \mathrm{~h}$, the ${ }^{211}$ At isotope is the subject of ongoing research in nuclear medicine since it decays either via emission of an $\alpha$ particle (to ${ }^{207} \mathrm{Bi}$ ) or via electron capture (to an extremely short-lived nuclide, ${ }^{211} \mathrm{Po}$, which undergoes further $\alpha$ decay). This makes ${ }^{211}$ At an ideal short-range radiation source for targeted $\alpha$ therapy in cancer treatment (see e.g. [5-10]).

\footnotetext{
* Corresponding author.

E-mail address: pascal.quinet@umons.ac.be (P. Quinet).
}

Because of its extreme rarity, the chemical and physical properties of astatine are almost totally unknown. Many of these have only been estimated based on its position in the periodic table, making of At the heaviest of halogens, i.e. the group of elements including fluorine, chlorine, bromine and iodine. As regards its electronic structure, the ground state of the astatine atom is $6 p^{52} \mathrm{P}_{3 / 2}$ while the lowest excited configurations are of the type $6 p^{4} n l$ (with $n l=7 s, 7 p, 6 d, 5 f, \ldots$ ). Nevertheless, only two excited levels $\left(6 \mathrm{p}^{4} 7 \mathrm{~s}{ }^{4} \mathrm{P}_{3 / 2,5 / 2}\right)$ were clearly classified until now [11], although some additional tentative identifications of higher-lying states were suggested from recent experiments [12,13]. As support to further experimental investigations of astatine, it is however useful to know the atomic structure of this element in the most complete possible way. Heavy neutral atoms such as At I represent also an interesting challenge for the theoretical calculations in view of their very complex atomic structures containing a large number of electrons.

In the present work, different theoretical models based on the pseudo-relativistic Hartree-Fock (HFR) approach have been used to compute the atomic structure and radiative parameters involving the lowest states within the $6 p^{5}, 6 p^{4} 7 s$ and $6 p^{4} 7 p$ configurations with the aim of classifying the spectral lines and the energy levels observed in recent experiments. A tentative identification of higher states belonging to $6 p^{4} 9 p$ and $6 p^{4} 10 p$ configurations is also reported. This study can be considered as an extension of our previous theoretical investigations dedicated to the atomic properties of some other short-lived radioelements such as technecium $(Z=43)[14]$, promethium $(Z=61)[15]$, polonium $(Z=84)$ 


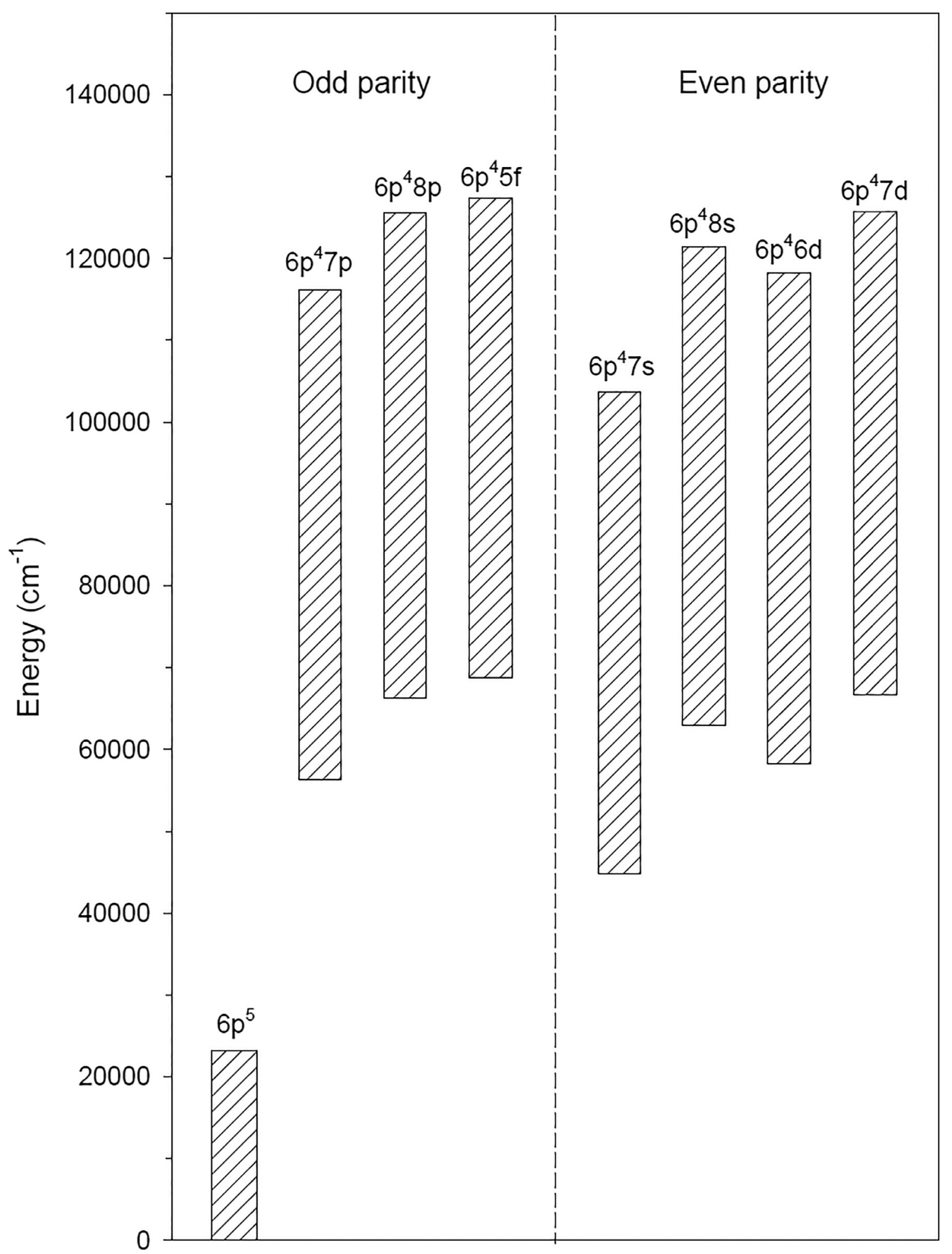

Fig. 1. Predicted locations of the lowest odd- and even-parity configurations in astatine atom.

[16], francium $(Z=87)$ [17], radium $(Z=88)$ [18], actinium $(Z=89)$ [18] and americium $(Z=95)$ [19].

\section{Available experimental data}

The first experimental study of the electronic structure of astatine atom was performed in 1964 by McLaughlin [11] who studied a $70 \mathrm{ng}$ sample of artificially produced radioactive isotopes and used a highly sensitive method of spectrographic detection of gases to observe the At I absorption spectrum. Two lines were recorded whose wavelengths were 216.225 and $224.401 \mathrm{~nm}$. These lines were tentatively assigned to the transitions from the ground state $6 \mathrm{p}^{5}{ }^{2} \mathrm{P}_{3 / 2}$ to the even-parity energy levels $6 \mathrm{p}^{4}\left({ }^{3} \mathrm{P}\right) 7 \mathrm{~s}{ }^{4} \mathrm{P}_{3 / 2}$ and $6 \mathrm{p}^{4}\left({ }^{3} \mathrm{P}\right) 7 \mathrm{~s} \quad{ }^{4} \mathrm{P}_{5 / 2}$ which were thus located at 46,233.6 and $44,549.3 \mathrm{~cm}^{-1}$, respectively. Fifty years later, these tentative assignments were confirmed by in-source laser resonance ionization 
spectroscopy studies of astatine isotopes using the resonance ionization laser ion source (RILIS) of the ISOLDE radioactive beam facility at CERN [12]. These latter experiments also allowed to measure the first ionization potential of astatine to $9.31751(8) \mathrm{eV}$, corresponding to $75,150.8(7) \mathrm{cm}^{-1}$. This successful measurement filled a long-standing gap in the Mendeleev's periodic table, since astatine was the last element in nature for which this fundamental property was unknown. In the same paper, it is interesting to note that the development of a three-colour ionization scheme, which required a search for higher lying atomic states, led to the observation of six new transitions between 710 and $915 \mathrm{~nm}$, connecting the $6 \mathrm{p}^{4}\left({ }^{3} \mathrm{P}\right) 7 \mathrm{~s}{ }^{4} \mathrm{P}_{3 / 2,5 / 2}$ levels to four higher odd-parity levels whose energies were established at 57,157.1, 57,267.8, 57,276.7 and $58,805.0 \mathrm{~cm}^{-1}$. Unfortunately, for these levels, no clear identification was given. More recently, at the Isotope Separator and Accelerator (ISAC) facility at TRIUMF, laser spectroscopy was performed to search for additional high lying bound states as well as for autoionizing resonances in astatine [13]. While no auto-ionizing resonances were measured in the investigated region, this experiment led however to the observation of four new odd-parity levels at $69,615.1,70,055.4,71,376.7$ and $71,708.7 \mathrm{~cm}^{-1}$, but, in this case also, no spectroscopic designation was reported.

\section{Low-lying energy levels in the $6 p^{5}, 6 p^{4} 7 s$ and $6 p^{4} 7 p$ configurations}

The first goal of our work was to clearly identify the energy levels observed between 0 and $58,805 \mathrm{~cm}^{-1}$ in previous experiments involving atomic astatine [12-13]. The computational procedure that we have used for modelling the atomic structure and calculating radiative transition parameters in At I is the pseudo-relativistic Hartree-Fock (HFR) method described in detail by Cowan [20]. In a first step, a very simple physical model including only the lowest configurations, namely $6 p^{5}, 6 p^{4} 7 p, 6 p^{4} 8 p, 6 p^{4} 5 f$ for the odd parity, and $6 p^{4} 7 s, 6 p^{4} 8 s, 6 p^{4} 6 d, 6 p^{4} 7 d$ for the even parity, unambiguously showed that the experimental energy levels located at $44,549.3$ and $46,233.6 \mathrm{~cm}^{-1}$ [11-13] belong to $6 \mathrm{p}^{4} 7 \mathrm{~s}$ while the levels observed at 57,157.1, 57,267.8, 57,276.7 and 58,805.0 $\mathrm{cm}^{-1}$ $[12,13]$ must be assigned to the $6 p^{4} 7 p$ configuration, as illustrated in Fig. 1.

In order to give these levels more complete spectroscopic designations, we investigated the effects of intravalence, core-valence and core-core electron correlations using six sets of HFR calculations (A, B, C, D, E and F), which are summarized in Table 1. As seen from this table, more and more electron correlation was progressively included when going from model $A$ to model $F$. More precisely, in calculation $A$, only valence correlation outside $6 s^{2} 6 p^{4}$ was considered while some single and double excitations from the $6 \mathrm{p}$ subshell were included in calculations $B$ and $C$, respectively. In calculation $\mathrm{D}$, core-valence correlation effects were added by including single excitations from the 6 s subshell while, in calculation $\mathrm{E}$, core-core electron correlation contributions were considered with double excitations from 6s. Finally, in model F, the core-polarization (CPOL) effects from the 78 electrons occupying the $1 s^{2} 2 s^{2} 2 p^{6} 3 s^{2} 3 p^{6} 3 d^{10} 4 s^{2} 4 p^{6} 4 d^{10} 4 f^{14} 5 s^{2} 5 p^{6} 5 d^{10}$ closed shells corresponding to the At VIII ionic core were estimated using a pseudo-potential and a correction to the dipole operator according to the HFR + CPOL procedure described in many of our previous papers (see e.g. [21-23]). These CPOL corrections depend on two paremeters, i.e. the electric dipole polarizability of the ionic core, $\alpha_{d}$, and the cut-off radius, $r_{c}$, which can be seen as a measure of the size of the ionic core. For the first parameter, a value of $\alpha_{d}=1.50 \mathrm{a}_{0}{ }^{3}$ was graphically extrapolated for At VIII from the theoretical values reported by Fraga et al. [24] for the first ions along the platinum isoelectronic sequence, i.e. $\alpha_{d}=13.36,8.10,5.47$ and $3.98 \mathrm{a}_{0}{ }^{3}$ for Au II, Hg III, Tl IV, and Pb V, respectively (see Fig. 2).

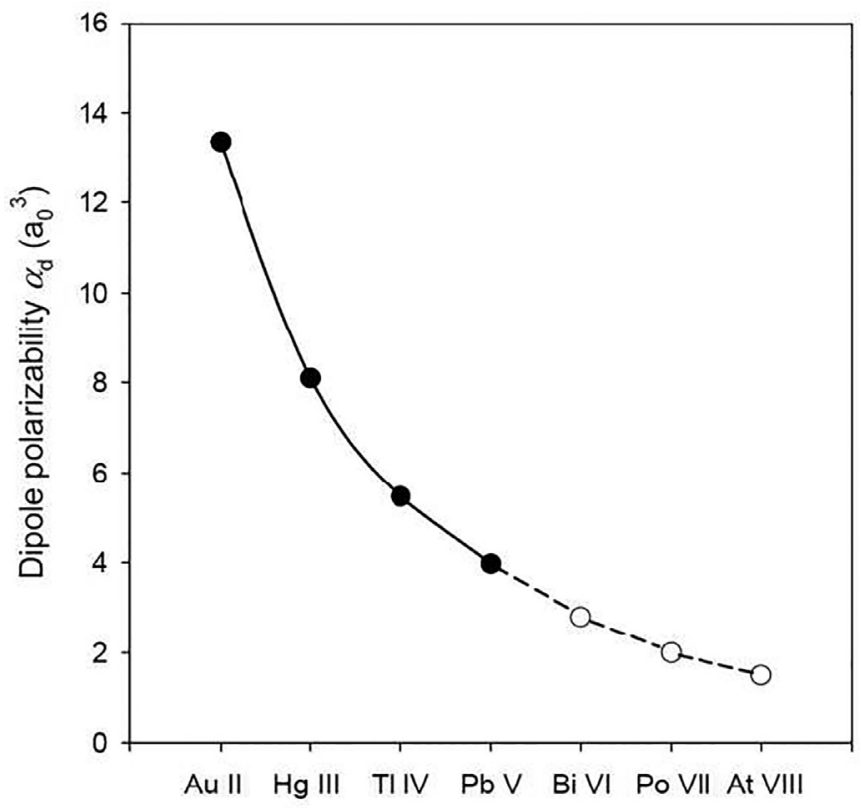

Fig. 2. Dipole polarizability along the platinum isoelectronic sequence. Full circles are theoretical values taken from [24]. Open circles connected with a dotted line represent extrapolated values.

The cut-off radius, $r_{c}$, was chosen to be equal to $1.12 \mathrm{a}_{0}$ which corresponds to the HFR average value $\langle r\rangle$ for the outermost core orbital $(5 d)$.

As already established in previous works [11-13], our calculations unsurprisingly confirmed the even-parity energy levels at $44,549.3$ and $46,233.6 \mathrm{~cm}^{-1}$ as corresponding to $6 \mathrm{p}^{4}\left({ }^{3} \mathrm{P}\right) 7 \mathrm{~s}{ }^{4} \mathrm{P}_{5 / 2}$ and $6 \mathrm{p}^{4}\left({ }^{3} \mathrm{P}\right) 7 \mathrm{~s}{ }^{4} \mathrm{P}_{3 / 2}$. Moreover, according to our calculations, the four odd-parity levels observed at 57,157.1, 57,267.8, 57,276.7 and $58,805.0 \mathrm{~cm}^{-1}[12-13]$ coincide almost perfectly with four theoretical energy values in the $6 p^{4} 7 p$ configuration with $J=5 / 2,7 / 2$, $1 / 2$ and $3 / 2$, respectively, all the other levels of this configuration appearing either too low (two levels around $56,000 \mathrm{~cm}^{-1}$ ) or too high (15 levels between 65,000 and $116,000 \mathrm{~cm}^{-1}$ ), whatever the HFR model used, as shown in Figs. 3 and 4. In Table 2, the lowest energy levels belonging to $6 p^{5}, 6 p^{4} 7 s$ and $6 p^{4} 7 p$, as computed with our most elaborate model, i.e. $\operatorname{HFR}(\mathrm{F})$, are compared with available experimental data. Let us mention that the best agreement between the calculated and experimental levels was found when considering configuration average energies $\left(E_{a v}\right)$ equal to $10,238,67,285$ and $79,061 \mathrm{~cm}^{-1}$ for $6 \mathrm{p}^{5}, 6 \mathrm{p}^{4} 7 \mathrm{~s}$ and $6 \mathrm{p}^{4} 7 \mathrm{p}$, respectively, while the corresponding ab initio $E_{a v}$-values were computed at $10,238,65,360$ and $76,727 \mathrm{~cm}^{-1}$. For each of the levels listed in Table 2, the first three eigenvector components are also given, allowing a complete identification for the first time.

It is worth noting that our calculations required that the $J$-values for the $6 p^{4} 7 \mathrm{p}$ levels at $57,267.8 \mathrm{~cm}^{-1}(J=7 / 2)$ and $57,276.7 \mathrm{~cm}^{-1}(J=1 / 2)$ be swapped in comparison with the predictions made in [13], i.e. $J=1 / 2$ and $7 / 2$, respectively. This is confirmed by the fact that, according to the laser excitation scheme illustrated in Fig. 1 of Ref. [12], the former level $\left(E=57,267.8 \mathrm{~cm}^{-1}\right)$ was populated through a transition from $6 \mathrm{p}^{4} 7 \mathrm{~s}{ }^{4} \mathrm{P}_{5 / 2}$ while the latter one $\left(E=57,276.7 \mathrm{~cm}^{-1}\right)$ was populated through a transition from $6 \mathrm{p}^{4} 7 \mathrm{~s}^{2} \mathrm{P}_{3 / 2}$, which prohibits the tentative J-assignements given in [13] because of the electric dipole radiation selection rule $\Delta J=0, \pm 1$. A further confirmation of our identifications of the levels in $6 p^{4} 7 p$ configuration of astatine is highlighted by the comparison with the $5 p^{4} 6 p$ configuration in the analogous iodine atom for which the corresponding energy levels were 
Table 1

Interacting configurations and core-polarization parameters retained in the HFR atomic structure calculations of neutral astatine.

\begin{tabular}{|c|c|c|c|c|c|c|}
\hline Type of calculation & A & B & C & $\mathrm{D}$ & $\mathrm{E}$ & $\mathrm{F}$ \\
\hline Odd configurations & $\begin{array}{l}6 s^{2} 6 p^{5} \\
6 s^{2} 6 p^{4} 7 p \\
6 s^{2} 6 p^{4} 5 f \\
6 s^{2} 6 p^{4} 6 f\end{array}$ & $\begin{array}{l}6 s^{2} 6 p^{5} \\
6 s^{2} 6 p^{4} 7 p \\
6 s^{2} 6 p^{4} 5 f \\
6 s^{2} 6 p^{4} 6 f \\
6 s^{2} 6 p^{3} 6 d^{2} \\
6 s^{2} 6 p^{3} 7 s^{2} \\
6 s^{2} 6 p^{3} 6 d 7 s \\
6 s^{2} 6 p^{3} 6 d 7 d \\
6 s^{2} 6 p^{3} 7 s 7 d \\
6 s^{2} 6 p^{3} 7 p 5 f \\
6 s^{2} 6 p^{3} 7 p 6 f\end{array}$ & $\begin{array}{l}6 s^{2} 6 p^{5} \\
6 s^{2} 6 p^{4} 7 p \\
6 s^{2} 6 p^{4} 5 f \\
6 s^{2} 6 p^{4} 6 f \\
6 s^{2} 6 p^{3} 6 d^{2} \\
6 s^{2} 6 p^{3} 7 s^{2} \\
6 s^{2} 6 p^{3} 6 d 7 s \\
6 s^{2} 6 p^{3} 6 d 7 d \\
6 s^{2} 6 p^{3} 7 s 7 d \\
6 s^{2} 6 p^{3} 7 p 5 f \\
6 s^{2} 6 p^{3} 7 p 6 f \\
6 s^{2} 6 p^{2} 7 p^{3} \\
6 s^{2} 6 p^{2} 6 d^{2} 7 p \\
6 s^{2} 6 p^{2} 7 s^{2} 7 p \\
6 s^{2} 6 p^{2} 6 d 7 s 7 p\end{array}$ & $\begin{array}{l}6 s^{2} 6 p^{5} \\
6 s^{2} 6 p^{4} 7 p \\
6 s^{2} 6 p^{4} 5 f \\
6 s^{2} 6 p^{4} 6 f \\
6 s^{2} 6 p^{3} 6 d^{2} \\
6 s^{2} 6 p^{3} 7 s^{2} \\
6 s^{2} 6 p^{3} 6 d 7 s \\
6 s^{2} 6 p^{3} 6 d 7 d \\
6 s^{2} 6 p^{3} 7 s 7 d \\
6 s^{2} 6 p^{3} 7 p 5 f \\
6 s^{2} 6 p^{3} 7 p 6 f \\
6 s^{2} 6 p^{2} 7 p^{3} \\
6 s^{2} 6 p^{2} 6 d^{2} 7 p \\
6 s^{2} 6 p^{2} 7 s^{2} 7 p \\
6 s^{2} 6 p^{2} 6 d 7 s 7 p \\
6 s 6 p^{5} 6 d \\
6 s 6 p^{5} 7 s \\
6 s 6 p^{4} 6 d 7 p \\
6 s 6 p^{4} 7 s 7 p\end{array}$ & $\begin{array}{l}6 s^{2} 6 p^{5} \\
6 s^{2} 6 p^{4} 7 p \\
6 s^{2} 6 p^{4} 5 f \\
6 s^{2} 6 p^{4} 6 f \\
6 s^{2} 6 p^{3} 6 d^{2} \\
6 s^{2} 6 p^{3} 7 s^{2} \\
6 s^{2} 6 p^{3} 6 d 7 s \\
6 s^{2} 6 p^{3} 6 d 7 d \\
6 s^{2} 6 p^{3} 7 s 7 d \\
6 s^{2} 6 p^{3} 7 p 5 f \\
6 s^{2} 6 p^{3} 7 p 6 f \\
6 s^{2} 6 p^{2} 7 p^{3} \\
6 s^{2} 6 p^{2} 6 d^{2} 7 p \\
6 s^{2} 6 p^{2} 7 s^{2} 7 p \\
6 s^{2} 6 p^{2} 6 d 7 s 7 p \\
6 s 6 p^{5} 6 d \\
6 s 6 p^{5} 7 s \\
6 s 6 p^{4} 6 d 7 p \\
6 s 6 p^{4} 7 s 7 p \\
6 p^{6} 7 p \\
6 p^{5} 6 d^{2} \\
6 p^{5} 7 s^{2} \\
6 p^{5} 7 p^{2} \\
6 p^{5} 6 d 7 s\end{array}$ & $\begin{array}{l}6 s^{2} 6 p^{5} \\
6 s^{2} 6 p^{4} 7 p \\
6 s^{2} 6 p^{4} 5 f \\
6 s^{2} 6 p^{4} 6 f \\
6 s^{2} 6 p^{3} 6 d^{2} \\
6 s^{2} 6 p^{3} 7 s^{2} \\
6 s^{2} 6 p^{3} 6 d 7 s \\
6 s^{2} 6 p^{3} 6 d 7 d \\
6 s^{2} 6 p^{3} 7 s 7 d \\
6 s^{2} 6 p^{3} 7 p 5 f \\
6 s^{2} 6 p^{3} 7 p 6 f \\
6 s^{2} 6 p^{2} 7 p^{3} \\
6 s^{2} 6 p^{2} 6 d^{2} 7 p \\
6 s^{2} 6 p^{2} 7 s^{2} 7 p \\
6 s^{2} 6 p^{2} 6 d 7 s 7 p \\
6 s 6 p^{5} 6 d \\
6 s 6 p^{5} 7 s \\
6 s 6 p^{4} 6 d 7 p \\
6 s 6 p^{4} 7 s 7 p \\
6 p^{6} 7 p \\
6 p^{5} 6 d^{2} \\
6 p^{5} 7 s^{2} \\
6 p^{5} 7 p^{2} \\
6 p^{5} 6 d 7 s\end{array}$ \\
\hline Even configurations & $\begin{array}{l}6 s^{2} 6 p^{4} 7 s \\
6 s^{2} 6 p^{4} 6 d \\
6 s^{2} 6 p^{4} 7 d\end{array}$ & $\begin{array}{l}6 s^{2} 6 p^{4} 7 s \\
6 s^{2} 6 p^{4} 6 d \\
6 s^{2} 6 p^{4} 7 d \\
6 s^{2} 6 p^{3} 6 d 7 p \\
6 s^{2} 6 p^{3} 7 s 7 p \\
6 s^{2} 6 p^{3} 6 d 5 f \\
6 s^{2} 6 p^{3} 6 d 6 f \\
6 s^{2} 6 p^{3} 7 s 5 f \\
6 s^{2} 6 p^{3} 7 s 6 f\end{array}$ & $\begin{array}{l}6 s^{2} 6 p^{4} 7 s \\
6 s^{2} 6 p^{4} 6 d \\
6 s^{2} 6 p^{4} 7 d \\
6 s^{2} 6 p^{3} 6 d 7 p \\
6 s^{2} 6 p^{3} 7 s 7 p \\
6 s^{2} 6 p^{3} 6 d 5 f \\
6 s^{2} 6 p^{3} 6 d 6 f \\
6 s^{2} 6 p^{3} 7 s 5 f \\
6 s^{2} 6 p^{3} 7 s 6 f \\
6 s^{2} 6 p^{2} 6 d^{3} \\
6 s^{2} 6 p^{2} 6 d^{2} 7 s \\
6 s^{2} 6 p^{2} 6 d 7 s^{2} \\
6 s^{2} 6 p^{2} 6 d 7 p^{2} \\
6 s^{2} 6 p^{2} 7 s 7 p^{2}\end{array}$ & $\begin{array}{l}6 s^{2} 6 p^{4} 7 s \\
6 s^{2} 6 p^{4} 6 d \\
6 s^{2} 6 p^{4} 7 d \\
6 s^{2} 6 p^{3} 6 d 7 p \\
6 s^{2} 6 p^{3} 7 s 7 p \\
6 s^{2} 6 p^{3} 6 d 5 f \\
6 s^{2} 6 p^{3} 6 d 6 f \\
6 s^{2} 6 p^{3} 7 s 5 f \\
6 s^{2} 6 p^{3} 7 s 6 f \\
6 s^{2} 6 p^{2} 6 d^{3} \\
6 s^{2} 6 p^{2} 6 d^{2} 7 s \\
6 s^{2} 6 p^{2} 6 d 7 s^{2} \\
6 s^{2} 6 p^{2} 6 d 7 p^{2} \\
6 s^{2} 6 p^{2} 7 s 7 p^{2} \\
6 s 6 p^{6} \\
6 s 6 p^{5} 7 p \\
6 s 6 p^{4} 6 d^{2} \\
6 s 6 p^{4} 7 s^{2} \\
6 s 6 p^{4} 7 p^{2} \\
6 s 6 p^{4} 6 d 7 s\end{array}$ & $\begin{array}{l}6 s^{2} 6 p^{4} 7 s \\
6 s^{2} 6 p^{4} 6 d \\
6 s^{2} 6 p^{4} 7 d \\
6 s^{2} 6 p^{3} 6 d 7 p \\
6 s^{2} 6 p^{3} 7 s 7 p \\
6 s^{2} 6 p^{3} 6 d 5 f \\
6 s^{2} 6 p^{3} 6 d 6 f \\
6 s^{2} 6 p^{3} 7 s 5 f \\
6 s^{2} 6 p^{3} 7 s 6 f \\
6 s^{2} 6 p^{2} 6 d^{3} \\
6 s^{2} 6 p^{2} 6 d^{2} 7 s \\
6 s^{2} 6 p^{2} 6 d 7 s^{2} \\
6 s^{2} 6 p^{2} 6 d 7 p^{2} \\
6 s^{2} 6 p^{2} 7 s 7 p^{2} \\
6 s 6 p^{6} \\
6 s 6 p^{5} 7 p \\
6 s 6 p^{4} 6 d^{2} \\
6 s 6 p^{4} 7 s^{2} \\
6 s 6 p^{4} 7 p^{2} \\
6 s 6 p^{4} 6 d 7 s \\
6 p^{6} 6 d \\
6 p^{6} 7 s \\
6 p^{5} 6 d 7 p \\
6 p^{5} 7 s 7 p\end{array}$ & $\begin{array}{l}6 s^{2} 6 p^{4} 7 s \\
6 s^{2} 6 p^{4} 6 d \\
6 s^{2} 6 p^{4} 7 d \\
6 s^{2} 6 p^{3} 6 d 7 p \\
6 s^{2} 6 p^{3} 7 s 7 p \\
6 s^{2} 6 p^{3} 6 d 5 f \\
6 s^{2} 6 p^{3} 6 d 6 f \\
6 s^{2} 6 p^{3} 7 s 5 f \\
6 s^{2} 6 p^{3} 7 s 6 f \\
6 s^{2} 6 p^{2} 6 d^{3} \\
6 s^{2} 6 p^{2} 6 d^{2} 7 s \\
6 s^{2} 6 p^{2} 6 d 7 s^{2} \\
6 s^{2} 6 p^{2} 6 d 7 p^{2} \\
6 s^{2} 6 p^{2} 7 s 7 p^{2} \\
6 s 6 p^{6} \\
6 s 6 p^{5} 7 p \\
6 s 6 p^{4} 6 d^{2} \\
6 s 6 p^{4} 7 s^{2} \\
6 s 6 p^{4} 7 p^{2} \\
6 s 6 p^{4} 6 d 7 s \\
6 p^{6} 6 d \\
6 p^{6} 7 s \\
6 p^{5} 6 d 7 p \\
6 p^{5} 7 s 7 p\end{array}$ \\
\hline Core-polarization parameters & & & & & & $\begin{array}{l}\alpha_{d}=1.50 \mathrm{a}_{0}{ }^{3} \\
r_{c}=1.12 \mathrm{a}_{0} \\
\text { (At VIII core) }\end{array}$ \\
\hline
\end{tabular}

Table 2

Classification of low-lying energy levels in $6 p^{5}, 6 p^{4} 7 \mathrm{~s}$ and $6 p^{4} 7 \mathrm{p}$ configurations of At I. Only the first three $L S$ components larger than $5 \%$ are given.

\begin{tabular}{lllllll}
\hline $\mathrm{E}_{\exp }\left(\mathrm{cm}^{-1}\right)^{\mathrm{a}}$ & $\mathrm{E}_{\text {calc }}\left(\mathrm{cm}^{-1}\right)^{\mathrm{b}}$ & $\Delta \mathrm{E}\left(\mathrm{cm}^{-1}\right)$ & $\mathrm{J}$ & 1st component (\%) & 2nd component (\%) & 3rd component (\%) \\
\hline 0.00 & 0 & 0 & $3 / 2$ & $996 \mathrm{p}^{5} \mathrm{P}$ & & \\
& 23,068 & & $1 / 2$ & $996 \mathrm{p}^{5} \mathrm{P}$ & & \\
$44,549.28$ & 44,653 & -104 & $5 / 2$ & $776 \mathrm{p}^{4}\left({ }^{3} \mathrm{P}\right) 7 \mathrm{~s}{ }^{4} \mathrm{P}$ & $216 \mathrm{p}^{4}\left({ }^{1} \mathrm{D}\right) 7 \mathrm{~s}^{2} \mathrm{D}$ & \\
$46,233.64$ & 46,128 & 106 & $3 / 2$ & $606 \mathrm{p}^{4}\left({ }^{3} \mathrm{P}\right) 7 \mathrm{~s}^{2} \mathrm{P}$ & $236 \mathrm{p}^{4}\left({ }^{1} \mathrm{D}\right) 7 \mathrm{~s}{ }^{2} \mathrm{D}$ & $156 \mathrm{p}^{4}\left({ }^{3} \mathrm{P}\right) 7 \mathrm{~s}{ }^{4} \mathrm{P}$ \\
& 55,910 & & $3 / 2$ & $396 \mathrm{p}^{4}\left({ }^{3} \mathrm{P}\right) 7 \mathrm{p}{ }^{4} \mathrm{P}$ & $206 \mathrm{p}^{4}\left({ }^{3} \mathrm{P}\right) 7 \mathrm{p}{ }^{4} \mathrm{~S}$ & $136 \mathrm{p}^{4}\left({ }^{1} \mathrm{D}\right) 7 \mathrm{p}{ }^{2} \mathrm{P}$ \\
$57,157.10$ & 57,051 & & $5 / 2$ & $326 \mathrm{p}^{4}\left({ }^{3} \mathrm{P}\right) 7 \mathrm{p}{ }^{4} \mathrm{D}$ & $276 \mathrm{p}^{4}\left({ }^{3} \mathrm{P}\right) 7 \mathrm{p}{ }^{4} \mathrm{P}$ & $196 \mathrm{p}^{4}\left({ }^{3} \mathrm{P}\right) 7 \mathrm{p}{ }^{2} \mathrm{D}$ \\
$57,267.80$ & 57,244 & 43 & $5 / 2$ & $416 \mathrm{p}^{4}\left({ }^{3} \mathrm{P}\right) 7 \mathrm{p}{ }^{2} \mathrm{D}$ & $376 \mathrm{p}^{4}\left({ }^{3} \mathrm{P}\right) 7 \mathrm{p}{ }^{4} \mathrm{P}$ & $126 \mathrm{p}^{4}\left({ }^{1} \mathrm{D}\right) 7 \mathrm{p}{ }^{2} \mathrm{D}$ \\
$57,276.70$ & 57,277 & 0 & $7 / 2$ & $776 \mathrm{p}^{4}\left({ }^{3} \mathrm{P}\right) 7 \mathrm{p}{ }^{4} \mathrm{D}$ & $226 \mathrm{p}^{4}\left({ }^{1} \mathrm{D}\right) 7 \mathrm{p}{ }^{2} \mathrm{~F}$ & \\
$58,805.00$ & 58,873 & -68 & $1 / 2$ & $366 \mathrm{p}^{4}\left({ }^{3} \mathrm{P}\right) 7 \mathrm{p}{ }^{2} \mathrm{~S}$ & $246 \mathrm{p}^{4}\left({ }^{1} \mathrm{D}\right) 7 \mathrm{p}{ }^{2} \mathrm{P}$ & $246 \mathrm{p}^{4}\left({ }^{3} \mathrm{P}\right) 7 \mathrm{p}{ }^{2} \mathrm{P}$ \\
\hline
\end{tabular}

a Experimental energy levels from [12]

b Calculated energy levels as obtained in the present work using the HFR(F) model. 


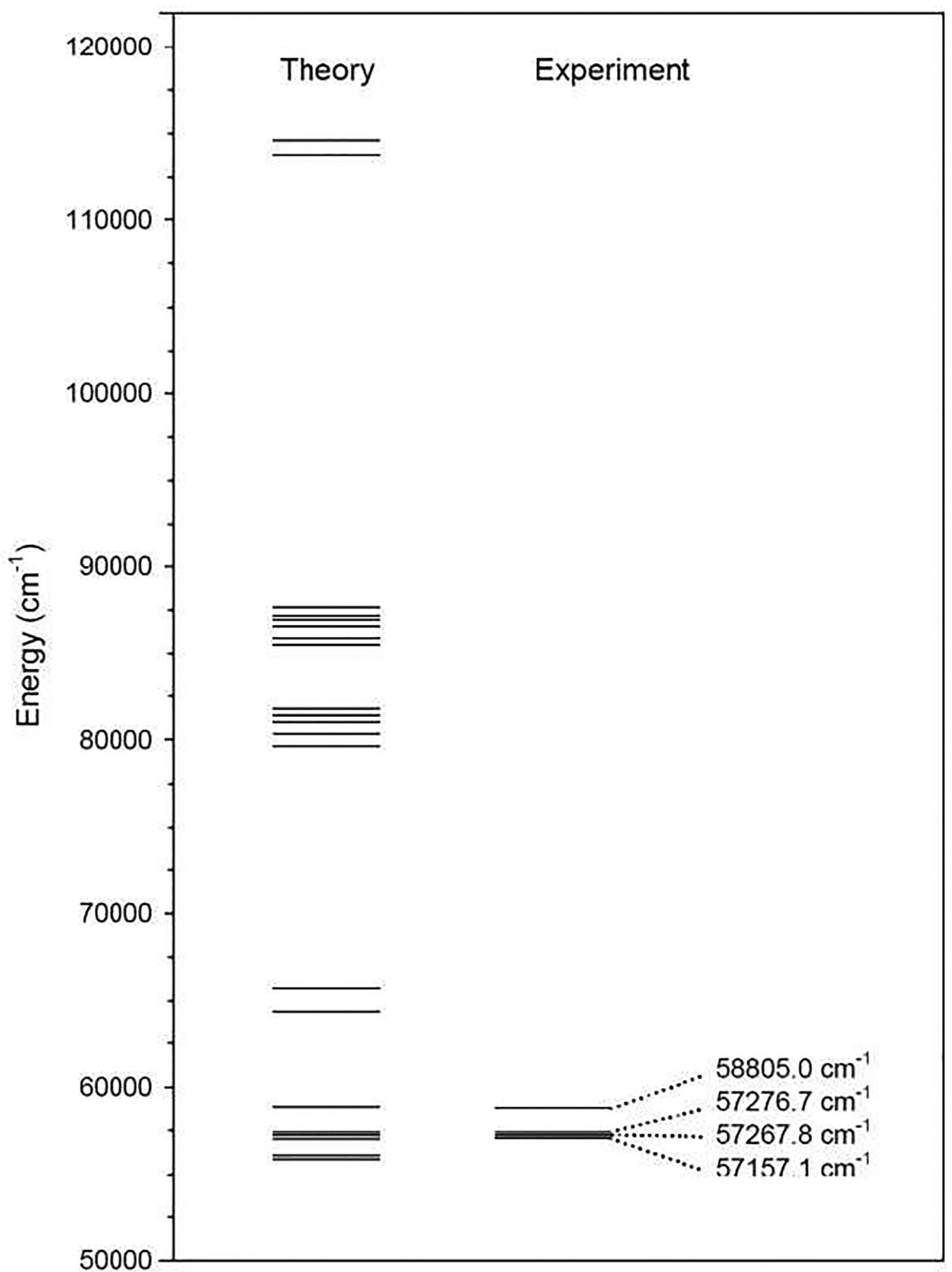

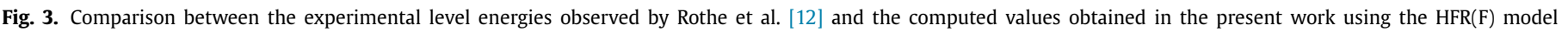
for the whole $6 p^{4} 7 p$ configuration in At I.

classified with the same order of the total angular momentum, i.e. $E=65,644.476 \mathrm{~cm}^{-1}(J=5 / 2), 65,669.988 \mathrm{~cm}^{-1}(J=7 / 2)$, $65,856.960 \mathrm{~cm}^{-1}(J=1 / 2)$ and $67,062.130 \mathrm{~cm}^{-1}(J=3 / 2)$, according to $[25]$.

The theoretical oscillator strengths, $\log g f$, and the weighted transition probabilities, $g A$, computed in the present work using the $\operatorname{HFR}(F)$ model are reported in Table 3 for the At I lines involving all the experimentally observed levels listed in Table 2. We note that all transitions listed in Table 3 are characterized by rather large oscillator strengths which not only justifies their detection in previous experimental studies [12-13] but also supplies an even more solid basis to our new energy level identifications. The influence of the different types of electron correlation on oscillator strength calculations was estimated through a detailed 


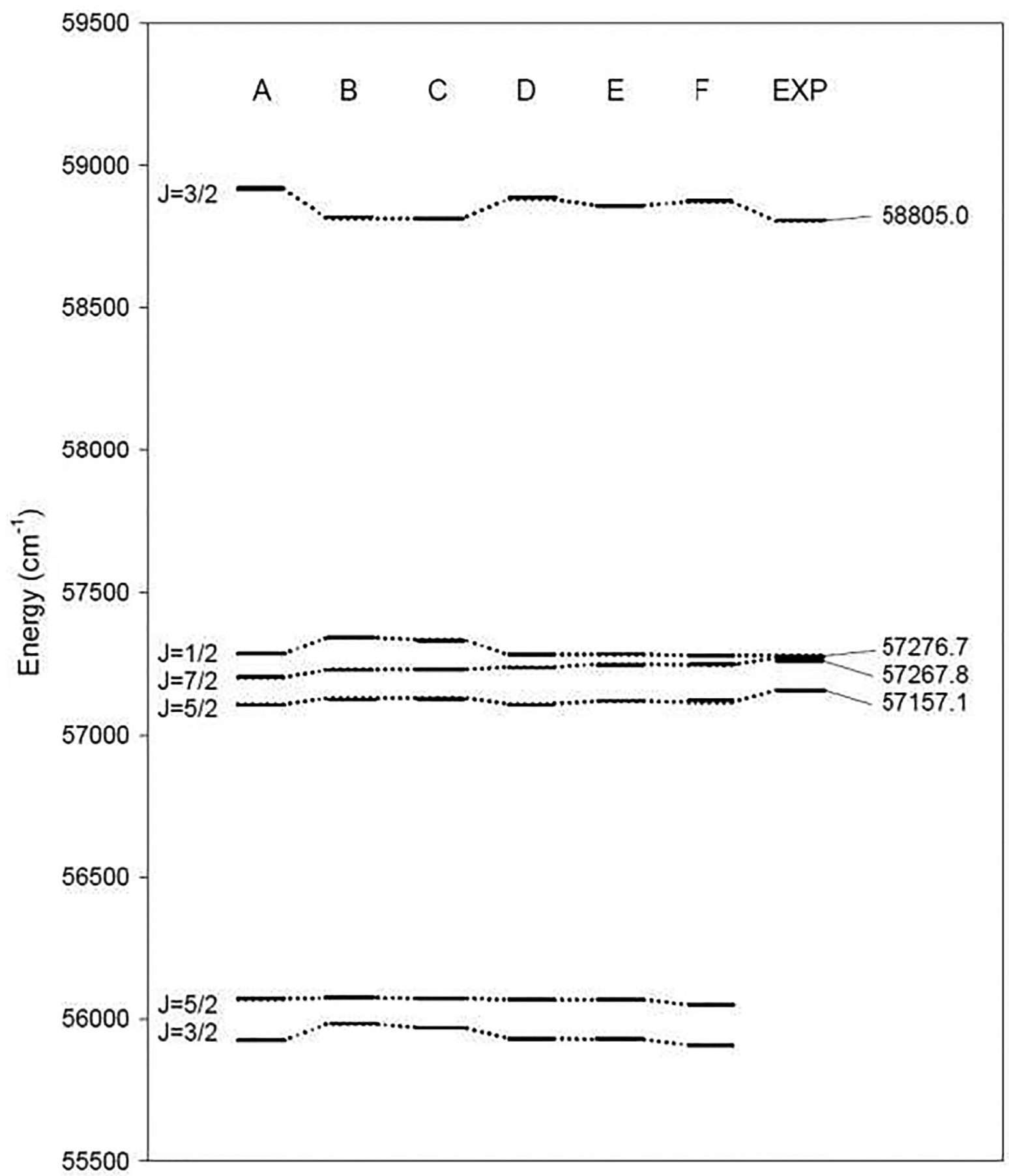

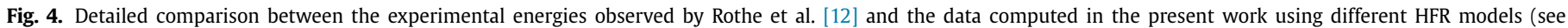
text) for the six lowest levels within the $6 p^{4} 7 p$ configuration of At I.

comparison between the results obtained using our different models, from HFR(A) to HFR(F). Such a comparison is illustrated in Fig. 5 where the ratios between the $g f$-values computed using our different models are plotted for all the At I lines listed in Table 3, numbered according to the order of appearance in the latter table. When looking at Fig. 5, one can observe that only single excitations from the $6 \mathrm{p}$ subshell (model $\mathrm{B}$ ) play a non negligible role in the calculation of $6 p^{5}-6 p^{4} 7 s$ and $6 p^{4} 7 s-6 p^{4} 7 p$ transition rates of interest while double excitations from $6 p$ as well as single and double excitations from $6 \mathrm{~s}$ (models $\mathrm{C}$ to $\mathrm{E}$ ) do not affect the oscillator strengths. Moreover, core-polarization effects from the At VIII $\left[1 \mathrm{~s}^{2} \ldots 5 \mathrm{~d}^{10}\right]$ ionic core (model $\mathrm{F}$ ) are rather small. This can be illustrated by the mean ratios of oscillator strengths obtained with two successive models for which the values were found to be equal to $g f(B) / g f(A)=0.94 \pm 0.08, g f(C) / g f(B)=1.00 \pm 0.01$, $g f(\mathrm{D}) / g f(\mathrm{C})=0.99 \pm 0.03, \quad g f(\mathrm{E}) / g f(\mathrm{D})=1.00 \pm 0.01 \quad$ and $\quad g f(\mathrm{~F}) /$ $g f(E)=0.99 \pm 0.03$ where the uncertainty represents the standard deviation. The same conclusions were already drawn when we considered the $6 p^{4}-6 p^{3} 7 s$ and $6 p^{3} 7 s-6 p^{3} 7 p$ transitions in the nearby atom of polonium [16] for which we highlihted on the other hand that the $6 \mathrm{p}^{4}-6 \mathrm{p}^{3} 6 \mathrm{~d}$ were more affected by the $6 \mathrm{p}^{2} \rightarrow 6 \mathrm{~d}^{2}$ double excitation and the $6 \mathrm{~s} \rightarrow 6 \mathrm{~d}$ single excitation. This outcome was also verified in the case of $6 p^{5}-6 p^{4} 6 d$ transitions in At I but, in view of the total lack of experimental 
Table 3

Calculated oscillator strengths ( $\log g f$ ) and transition probabilities $(g A)$ for spectral lines connecting the experimentally known levels in $6 \mathrm{p}^{5}, 6 \mathrm{p}^{4} 7 \mathrm{~s}$ and $6 \mathrm{p}^{4} 7 \mathrm{p}$ configurations in At I.

\begin{tabular}{|c|c|c|c|c|c|c|c|}
\hline$\lambda(\mathrm{nm})^{\mathrm{a}}$ & $\lambda(\mathrm{nm})^{\mathrm{b}}$ & Lower level & & Upper level & & $\log g f^{d}$ & $g A\left(\mathrm{~s}^{-1}\right)^{\mathrm{d}}$ \\
\hline 216.225 & 216.72 & 0.00 & $\mathrm{~J}=3 / 2($ odd $)$ & $46,233.64$ & $\mathrm{~J}=3 / 2$ (even) & -0.05 & $1.25 \mathrm{E}+9$ \\
\hline 224.401 & 223.88 & 0.00 & $\mathrm{~J}=3 / 2$ (odd) & $44,549.28$ & $\mathrm{~J}=5 / 2$ (even) & -0.90 & $1.67 \mathrm{E}+8$ \\
\hline 701.279 & 703.04 & $44,549.28$ & $\mathrm{~J}=5 / 2$ (even) & $58,805.00$ & $\mathrm{~J}=3 / 2($ odd $)$ & -0.58 & $3.54 \mathrm{E}+7$ \\
\hline 786.039 & 794.00 & $44,549.28$ & $\mathrm{~J}=5 / 2$ (even) & $57,267.80$ & $\mathrm{~J}=7 / 2($ odd $)$ & 0.53 & $3.61 \mathrm{E}+8$ \\
\hline 792.940 & 802.28 & $44,549.28$ & $\mathrm{~J}=5 / 2$ (even) & $57,157.10$ & $\mathrm{~J}=5 / 2($ odd $)$ & 0.00 & $1.03 \mathrm{E}+8$ \\
\hline 795.240 & 784.41 & $46,233.64$ & $\mathrm{~J}=3 / 2$ (even) & $58,805.00$ & $\mathrm{~J}=3 / 2($ odd $)$ & 0.05 & $1.22 \mathrm{E}+8$ \\
\hline 905.298 & 896.69 & $46,233.64$ & $\mathrm{~J}=3 / 2$ (even) & $57,276.70$ & $\mathrm{~J}=1 / 2($ odd $)$ & -0.06 & $7.32 \mathrm{E}+7$ \\
\hline 915.210 & 910.00 & $46,233.64$ & $\mathrm{~J}=3 / 2$ (even) & $57,157.10$ & $\mathrm{~J}=5 / 2($ odd $)$ & 0.20 & $1.28 \mathrm{E}+8$ \\
\hline
\end{tabular}

a Air wavelengths deduced from the experimental energy levels [12].

b Air wavelengths deduced from the calculated energy levels using the HFR(F) model.

${ }^{c}$ Experimental energy level values (see Table 2 for the complete spectroscopic designations).

d Calculated values as obtained in the present work using the HFR(F) model.

Table 4

Comparison between theoretical and experimental energies for odd-parity levels in the region $69,000-72,000 \mathrm{~cm}^{-1}$ and tentative identification of levels belonging to the $6 p^{4} 9 p$ and $6 p^{4} 10 p$ configurations in At I. Calculated oscillator strengths (log $g$ ) for spectral lines connecting those levels to the two experimentally known $6 p^{4} 7 \mathrm{~s}$ levels are also given.

\begin{tabular}{|c|c|c|c|c|c|}
\hline $\mathrm{E}_{\exp }{ }^{\mathrm{a}}\left(\mathrm{cm}^{-1}\right)$ & $\mathrm{E}_{\text {calc }}{ }^{\mathrm{b}}\left(\mathrm{cm}^{-1}\right)$ & $\mathrm{J}$ & Composition in $L S$-coupling ${ }^{\mathrm{C}}(\%)$ & $\begin{array}{l}\text { Calculated log } g f \\
44,549.2\left({ }^{4} \mathrm{P}_{5 / 2}\right)\end{array}$ & $\begin{array}{l}\text { alues for transitions to lower } 6 \mathrm{p}^{4} 7 \mathrm{~s} \text { levels }{ }^{\mathrm{b}} \\
46,233.6\left({ }^{2} \mathrm{P}_{3 / 2}\right)\end{array}$ \\
\hline & 69,624 & $3 / 2$ & $366 p^{4}\left({ }^{3} P\right) 9 p{ }^{4} P+236 p^{4}\left({ }^{3} P\right) 9 p{ }^{4} S+126 p^{4}\left({ }^{1} D\right) 9 p{ }^{2} P$ & -1.96 & -2.62 \\
\hline & 69,637 & $5 / 2$ & $326 \mathrm{p}^{4}\left({ }^{3} \mathrm{p}\right) 9 \mathrm{p}{ }^{4} \mathrm{D}+246 \mathrm{p}^{4}\left({ }^{3} \mathrm{p}\right) 9 \mathrm{p}{ }^{4} \mathrm{P}+226 \mathrm{p}^{4}\left({ }^{3} \mathrm{P}\right) 9 \mathrm{p}{ }^{2} \mathrm{D}$ & -1.99 & -2.02 \\
\hline & 69,794 & $5 / 2$ & $436 p^{4}\left({ }^{3} p\right) 9 p{ }^{2} D+356 p^{4}\left({ }^{3} p\right) 9 p{ }^{4} p+136 p^{4}\left({ }^{1} D\right) 9 p{ }^{2} D$ & -2.36 & -1.83 \\
\hline \multirow[t]{2}{*}{$69,615.1$} & 69,806 & $7 / 2$ & $786 \mathrm{p}^{4}\left({ }^{3} \mathrm{p}\right) 9 \mathrm{p}{ }^{4} \mathrm{D}+226 \mathrm{p}^{4}\left({ }^{1} \mathrm{D}\right) 9 \mathrm{p}{ }^{2} \mathrm{~F}$ & -1.58 & - \\
\hline & 69,824 & $1 / 2$ & $426 \mathrm{p}^{4}\left({ }^{3} \mathrm{p}\right) 9 \mathrm{p}{ }^{2} \mathrm{~S}+226 \mathrm{p}^{4}\left({ }^{1} \mathrm{D}\right) 9 \mathrm{p}{ }^{2} \mathrm{P}+226 \mathrm{p}^{4}\left({ }^{3} \mathrm{p}\right) 9 \mathrm{p}{ }^{2} \mathrm{P}$ & - & -2.14 \\
\hline \multirow[t]{3}{*}{$70,055.4$} & 70,057 & $3 / 2$ & $446 p^{4}\left({ }^{3} \mathrm{P}\right) 9 \mathrm{p}{ }^{2} \mathrm{P}+206 \mathrm{p}^{4}\left({ }^{3} \mathrm{P}\right) 9 \mathrm{p}{ }^{4} \mathrm{~S}+126 \mathrm{p}^{4}\left({ }^{1} \mathrm{D}\right) 9 \mathrm{p}{ }^{2} \mathrm{D}$ & -2.09 & -1.39 \\
\hline & 71,464 & $3 / 2$ & $356 \mathrm{p}^{4}\left({ }^{3} \mathrm{P}\right) 10 \mathrm{p}{ }^{4} \mathrm{P}+236 \mathrm{p}^{4}\left({ }^{3} \mathrm{P}\right) 10 \mathrm{p}{ }^{4} \mathrm{~S}+126 \mathrm{p}^{4}\left({ }^{1} \mathrm{D}\right) 10 \mathrm{p}{ }^{2} \mathrm{P}$ & -2.38 & -3.00 \\
\hline & 71,474 & $5 / 2$ & $326 p^{4}\left({ }^{3} \mathrm{P}\right) 10 \mathrm{p}{ }^{4} \mathrm{D}+236 \mathrm{p}^{4}\left({ }^{3} \mathrm{P}\right) 10 \mathrm{p}{ }^{4} \mathrm{P}+226 \mathrm{p}^{4}\left({ }^{3} \mathrm{P}\right) 10 \mathrm{p}{ }^{2} \mathrm{D}$ & -2.35 & -2.38 \\
\hline \multirow[t]{3}{*}{$71,376.7$} & 71,560 & $5 / 2$ & $436 p^{4}\left({ }^{3} p\right) 10 p{ }^{2} D+356 p^{4}\left({ }^{3} P\right) 10 p{ }^{4} p+136 p^{4}\left({ }^{1} D\right) 10 p{ }^{2} D$ & -2.77 & -2.22 \\
\hline & 71,566 & $7 / 2$ & $786 \mathrm{p}^{4}\left({ }^{3} \mathrm{P}\right) 10 \mathrm{p}{ }^{4} \mathrm{D}+226 \mathrm{p}^{4}\left({ }^{1} \mathrm{D}\right) 10 \mathrm{p}{ }^{2} \mathrm{~F}$ & -1.97 & - \\
\hline & 71,573 & $1 / 2$ & $436 p^{4}\left({ }^{3} \mathrm{P}\right) 10 \mathrm{p}^{2} \mathrm{~S}+226 \mathrm{p}^{4}\left({ }^{1} \mathrm{D}\right) 10 \mathrm{p}^{2} \mathrm{P}+226 \mathrm{p}^{4}\left({ }^{3} \mathrm{P}\right) 10 \mathrm{p}{ }^{2} \mathrm{P}$ & - & -2.54 \\
\hline $71,708.7$ & 71,709 & $3 / 2$ & $446 p^{4}\left({ }^{3} \mathrm{P}\right) 10 \mathrm{p}{ }^{2} \mathrm{P}+206 \mathrm{p}^{4}\left({ }^{3} \mathrm{P}\right) 10 \mathrm{p}{ }^{4} \mathrm{~S}+126 \mathrm{p}^{4}\left({ }^{1} \mathrm{D}\right) 10 \mathrm{p}{ }^{2} \mathrm{D}$ & -2.38 & -1.68 \\
\hline
\end{tabular}

a Experimental energy level values taken from [13]

b HFR calculations (this work).

c Only the first three components larger than $5 \%$ are given

observation concerning the $6 \mathrm{p}^{4} 6 \mathrm{~d}$ configuration in this atom, the corresponding spectral lines are not reported in the present paper.

\section{New identification of energy levels in the $6 p^{4} 9 p$ and $6 p^{4} 10 p$ configurations}

Very recently, at the Isotope Separator and Accelerator (ISAC) facility at TRIUMF, laser spectroscopy was carried out to search for additional high lying bound states as well as for auto-ionizing resonances in astatine [13]. In particular, in the latter study, starting from the $6 \mathrm{p}^{4}\left({ }^{3} \mathrm{P}\right) 7 \mathrm{~s}{ }^{4} \mathrm{P}_{3 / 2}$ level at $46,233.64 \mathrm{~cm}^{-1}$, a scanning laser was used to search for odd-parity bound states near the ionization potential, more precisely between 69,800 and $73,800 \mathrm{~cm}^{-1}$. This led to the observation of three levels at 70,055.4, 71,376.7 and $71,708.7 \mathrm{~cm}^{-1}$. In addition, another brief scan from the $6 \mathrm{p}^{4}\left({ }^{3} \mathrm{P}\right) 7 \mathrm{~s}$ ${ }^{4} \mathrm{P}_{5 / 2}$ level at $44,549.28 \mathrm{~cm}^{-1}$ could be performed, revealing one further state of odd parity at $69,615.1 \mathrm{~cm}^{-1}$. Unfortunately, no attempt of spectroscopic designation was reported in [13]. As they were both populated from $6 \mathrm{p}^{4}\left({ }^{3} \mathrm{P}\right) 7 \mathrm{~s}$, the only certainty is that these four levels must belong to $6 \mathrm{p}^{4} n \mathrm{p}$ configurations, and most likely to $6 \mathrm{p}^{4}\left({ }^{3} \mathrm{P}\right) n \mathrm{p}$ sub configurations.

When performing a rather simple HFR calculation, including only $6 \mathrm{p}^{5}$ and $6 \mathrm{p}^{4} n \mathrm{p}(n=7-12)$, in which all the average energies of $6 p^{4} n p(n=8-12)$ configurations were shifted by the same amount as the one needed to reproduce at best the four experimental $6 p^{4} 7 p$ energy levels (see Section 3 hereabove), it became obvious that the levels at $69,615.1$ and $70,055.4 \mathrm{~cm}^{-1}$ had to belong to the $6 p^{4}\left({ }^{3} \mathrm{P}\right) 9 \mathrm{p}$ sub configuration while those observed at $71,376.7$ and $71,708.7 \mathrm{~cm}^{-1}$ had to belong to $6 \mathrm{p}^{4}\left({ }^{3} \mathrm{P}\right) 10 \mathrm{p}$. This is shown in Fig. 6 where the calculated energy levels of $6 p^{4} n p$ configurations are compared with the experimental observations, A more detailed investigation of $6 p^{4}\left({ }^{3} \mathrm{P}\right) 9 \mathrm{p}$ and $6 \mathrm{p}^{4}\left({ }^{3} \mathrm{P}\right) 10 \mathrm{p}$ showed actually that each of these sub configurations gives rise to a group of six close computed levels around 69,800 and $71,600 \mathrm{~cm}^{-1}$, respectively. It turned out to be difficult to use these theoretical energies to definitely identify the observed levels to such an extent that our reasoning could only be based on hypotheses. In this respect, a credible hypothesis was to assume that the most intense transitions from $6 \mathrm{p}^{4}\left({ }^{3} \mathrm{P}\right) 7 \mathrm{~s}^{2} \mathrm{P}_{3 / 2}\left(E=46,233.64 \mathrm{~cm}^{-1}\right)$ were used to populate the $6 p^{4} 9 p$ and $6 p^{4} 10 p$ levels in the laser spectroscopy experiment [13]. From our calculations, two transitions clearly appeared to have $g f$-values (at least a factor of 3 ) larger than the others, namely $6 \mathrm{p}^{4}\left({ }^{3} \mathrm{P}\right) 7 \mathrm{~s}{ }^{2} \mathrm{P}_{3 / 2}-6 \mathrm{p}^{4}\left({ }^{3} \mathrm{P}\right) 9 \mathrm{p}{ }^{2} \mathrm{P}_{3 / 2} \quad(\log g f=-1.39)$ and $6 \mathrm{p}^{4}\left({ }^{3} \mathrm{P}\right) 7 \mathrm{~s}{ }^{2} \mathrm{P}_{3 / 2}-6 \mathrm{p}^{4}\left({ }^{3} \mathrm{P}\right) 10 \mathrm{p}{ }^{2} \mathrm{P}_{3 / 2}$ (log $\left.g f=-1.68\right)$. In both cases, the upper level of the transition corresponds to the highest level among the group of six mentioned above, which implies that the level observed at $70,055.4 \mathrm{~cm}^{-1}$ should be identified as $\left.6 \mathrm{p}^{4}{ }^{3} \mathrm{P}\right) 9 \mathrm{p}^{2} \mathrm{P}_{3 / 2}$ while the one observed at $71,708.7 \mathrm{~cm}^{-1}$ should be $6 p^{4}\left({ }^{3} \mathrm{P}\right) 10 \mathrm{p}{ }^{2} \mathrm{P}_{3 / 2}$. The situation was much less clear for the other two experimental levels (at 69,615.1 and 71,376.7 $\mathrm{cm}^{-1}$ ) since they appeared to be reachable by different transitions from $6 p^{4}\left({ }^{3} \mathrm{P}\right) 7 \mathrm{~s}$ ${ }^{4} \mathrm{P}_{5 / 2}$ (for the former level) and $6 \mathrm{p}^{4}\left({ }^{3} \mathrm{P}\right) 7 \mathrm{~s}{ }^{2} \mathrm{P}_{3 / 2}$ (for the latter one) with comparable oscillator strengths. However, if we consider the largest of these oscillator strengths, i.e. $\log g f=-1.58$ and -2.21 , respectively, the level at $69,615.1 \mathrm{~cm}^{-1}$ might be tentatively attributed to $6 \mathrm{p}^{4}\left({ }^{3} \mathrm{P}\right) 9 \mathrm{p}{ }^{4} \mathrm{D}_{7 / 2}$ while the one at $71,376.7 \mathrm{~cm}^{-1}$ might correspond to $6 \mathrm{p}^{4}\left({ }^{3} \mathrm{P}\right) 10 \mathrm{p}{ }^{2} \mathrm{D}_{5 / 2}$, this latter level being populated from the lower $6 \mathrm{p}^{4}\left({ }^{3} \mathrm{P}\right) 7 \mathrm{~s}{ }^{4} \mathrm{P}_{3 / 2}$ level at $46,233.64 \mathrm{~cm}^{-1}$, according to [13]. A summary of this discussion is given in Table 4 in which a 

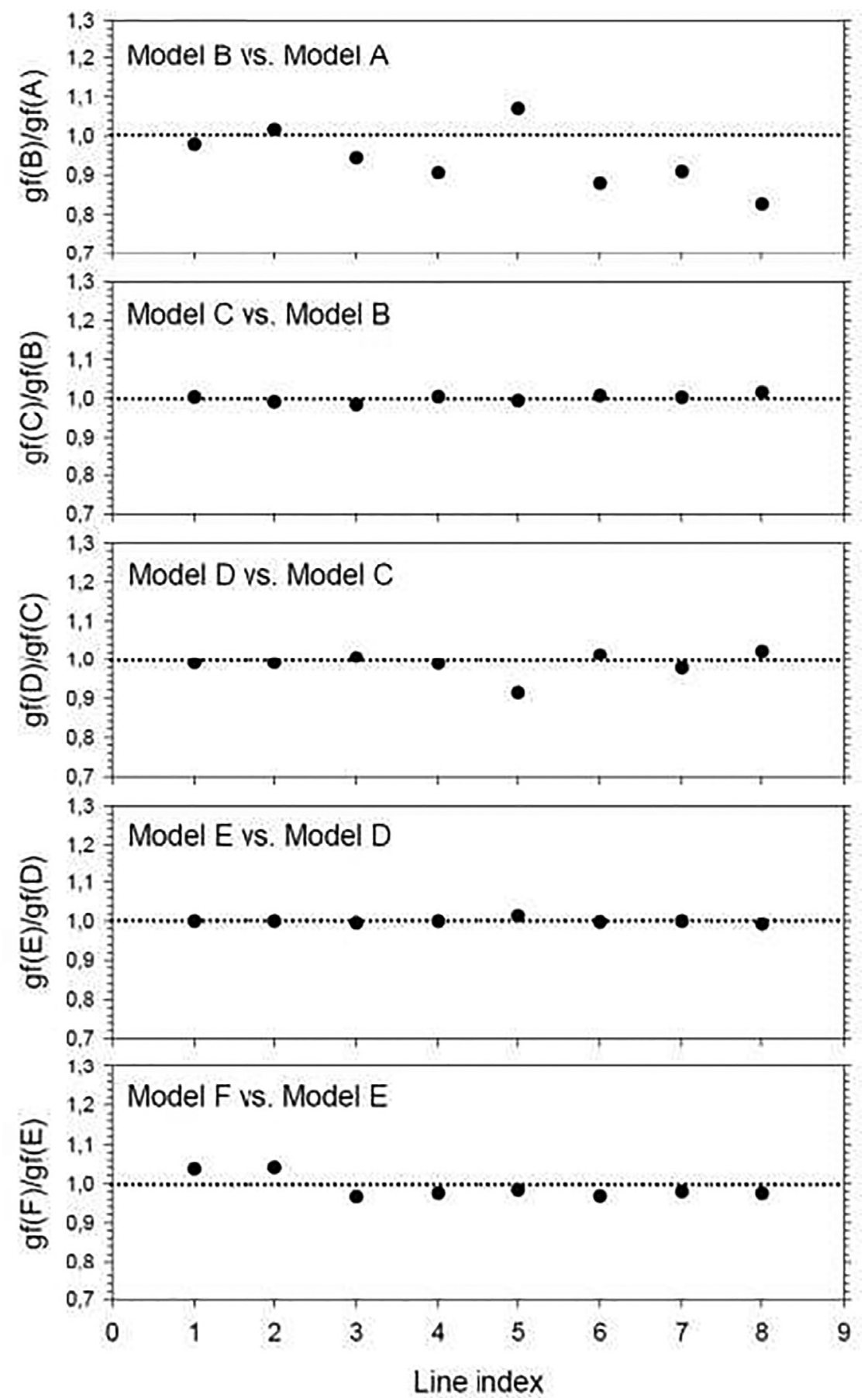

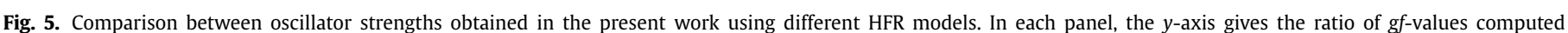

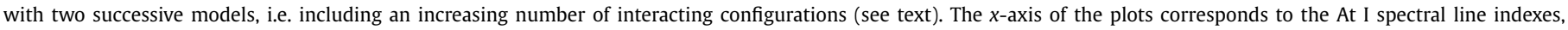
which are assigned according to the order of appearance in Table 3 (i.e. by increasing wavelengths).

comparison theory - observation is given for the levels of interest in $6 \mathrm{p}^{4} 9 \mathrm{p}$ and $6 \mathrm{p}^{4} 10 \mathrm{p}$ together with the calculated log gf-values for the corresponding $6 p^{4} 7 s-6 p^{4} 9 p$ and $6 p^{4} 7 s-6 p^{4} 10 p$ transitions.

\section{Predicted energy levels belonging to $6 p^{4} n p$ and $6 p^{4} n d$ Rydberg series}

When scanning the ionization path from the $6 \mathrm{p}^{4}\left({ }^{3} \mathrm{P}\right) 7 \mathrm{p}^{2} \mathrm{P}_{3 / 2}$ level at $58,805.0 \mathrm{~cm}^{-1}$ with their laser system in the visible range $(216-795 \mathrm{~nm})$ to probe the optical spectrum of astatine near the ionization threshold, Rothe et al. [12] observed more than 30
Rydberg levels. Following the trends of quantum defects of $n \mathrm{~d}$ and $n$ s series of the analogous bromine and iodine atoms, the assumption of a $n d$ series allowed the latter authors to assign principal quantum numbers to the observed Rydberg states. Using the quantum defect formula

$E_{n l}=E_{i o n}-\frac{R}{\left(n-\delta_{n l}\right)^{2}}$

the peak positions $E_{n l}$ were fitted with the ionization energy, $E_{\text {ion }}$, and the quantum defect, $\delta_{\mathrm{nl}}$, as free parameters. This approach led to $E_{\text {ion }}=75,150.8 \pm 0.7 \mathrm{~cm}^{-1}$ and $\delta_{\mathrm{nl}}=3.16$. However, no $6 \mathrm{p}^{4} n \mathrm{~d}$ energy level values were explicitly given in the paper of Rothe 


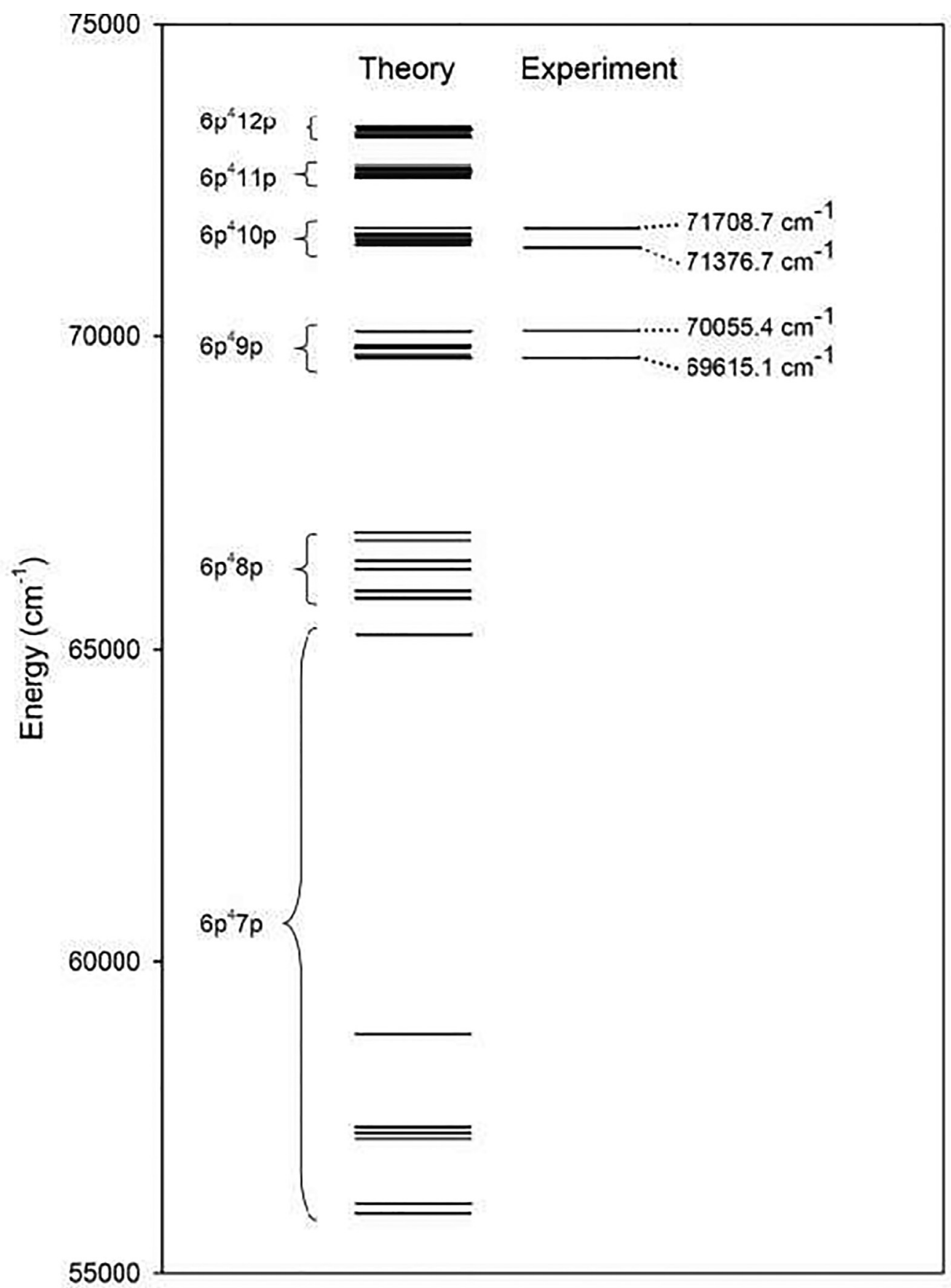

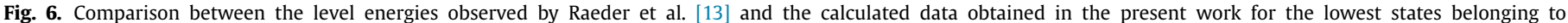
odd-parity $6 \mathrm{p}^{4} n \mathrm{p}(n=7-12)$ configurations of At I.

et al. [12]. In our work, these energies were computed for principal quantum numbers from $n=8$ to $n=50$ using, in addition to the aforementioned ionization energy and quantum defect values, a reduced-mass Rydberg constant corresponding to the mean value obtained when considering the astatine isotopes from ${ }^{191} \mathrm{At}$ to ${ }^{229}$ At, i.e. $R=109,737.03 \pm 0.03 \mathrm{~cm}^{-1}$. The results obtained are listed in Table 5 . The uncertainties $\varepsilon\left(E_{n l}\right)$ affecting our calculated energy level values were estimated using the well-known error propagation formula:

$\varepsilon\left(E_{n l}\right)=\sqrt{\left(\frac{\partial E_{n l}}{\partial E_{i o n}}\right)^{2} \varepsilon\left(E_{i o n}\right)^{2}+\left(\frac{\partial E_{n l}}{\partial R}\right)^{2} \varepsilon(R)^{2}+\left(\frac{\partial E_{n l}}{\partial \delta_{n l}}\right)^{2} \varepsilon\left(\delta_{n l}\right)^{2}}$

where the uncertainties corresponding to the ionization energy and the Rydberg constant were taken as being $\varepsilon\left(E_{\text {ion }}\right)=0.7 \mathrm{~cm}^{-1}$ and $\varepsilon(R)=0.03 \mathrm{~cm}^{-1}$, as mentioned above, and where the uncertainty affecting the quantum defect was assumed to be $\varepsilon\left(\delta_{\mathrm{nl}}\right)=0.05$. Note that, in Table 5 , we made the assumption of a ${ }^{2} D_{5 / 2}$ level along the $6 p^{4}\left({ }^{3} \mathrm{P}\right) n$ d series since this level corresponds by far to the most intense transition from the members of each $6 \mathrm{p}^{4}\left({ }^{3} \mathrm{P}\right) n$ d sub configuration to the $6 \mathrm{p}^{4}\left({ }^{3} \mathrm{P}\right) 7 \mathrm{p}{ }^{2} \mathrm{P}_{3 / 2}$ level at $58,805.0 \mathrm{~cm}^{-1}$, according to our calculations.

A similar approach could also be used for estimating the energies of the ${ }^{2} \mathrm{P}_{3 / 2}$ states along the $6 \mathrm{p}^{4}\left({ }^{3} \mathrm{P}\right) n \mathrm{p}$ Rydberg series from the levels at $70,055.4 \mathrm{~cm}^{-1}$ and $71,708.7 \mathrm{~cm}^{-1}$ that we idenfified as $6 \mathrm{p}^{4}\left({ }^{3} \mathrm{P}\right) 9 \mathrm{p}{ }^{2} \mathrm{P}_{3 / 2}$ and $6 \mathrm{p}^{4}\left({ }^{3} \mathrm{P}\right) 10 \mathrm{p}{ }^{2} \mathrm{P}_{3 / 2}$, respectively (see Section 4). For these two levels, we found the same quantum defect value, $\delta_{\mathrm{nl}}=4.36$, which allowed us to compute the energies 
Table 5

Predicted energies for the ${ }^{2} \mathrm{D}_{5 / 2}$ levels along the $6 \mathrm{p}^{4}\left({ }^{3} \mathrm{P}\right) n \mathrm{~d}$ Rydberg series from $n=8$ to $n=50$, as obtained using the quantum defect formula with $E_{\text {ion }}=75,150.8 \pm 0.7 \mathrm{~cm}^{-1}$, $R=109,737.03 \pm 0.03 \mathrm{~cm}^{-1}$ and $\delta_{n l}=3.15 \pm 0.05$.

\begin{tabular}{|c|c|}
\hline$n$ & $\mathrm{E}\left(\mathrm{cm}^{-1}\right)$ \\
\hline 8 & $70,466 \pm 97$ \\
\hline 9 & $71,933 \pm 55$ \\
\hline 10 & $72,805 \pm 34$ \\
\hline 11 & $73,365 \pm 23$ \\
\hline 12 & $73,747 \pm 16$ \\
\hline 13 & $74,017 \pm 12$ \\
\hline 14 & $74,217 \pm 9$ \\
\hline 15 & $74,368 \pm 7$ \\
\hline 16 & $74,485 \pm 5$ \\
\hline 17 & $74,578 \pm 4$ \\
\hline 18 & $74,653 \pm 3$ \\
\hline 19 & $74,713 \pm 3$ \\
\hline 20 & $74,764 \pm 2$ \\
\hline 21 & $74,806 \pm 2$ \\
\hline 22 & $74,842 \pm 2$ \\
\hline 23 & $74,872 \pm 2$ \\
\hline 24 & $74,898 \pm 1$ \\
\hline 25 & $74,921 \pm 1$ \\
\hline 26 & $74,940 \pm 1$ \\
\hline 27 & $74,958 \pm 1$ \\
\hline 28 & $74,973 \pm 1$ \\
\hline 29 & $74,986 \pm 1$ \\
\hline 30 & $74,998 \pm 1$ \\
\hline 31 & $75,009 \pm 1$ \\
\hline 32 & $75,019 \pm 1$ \\
\hline 33 & $75,028 \pm 1$ \\
\hline 34 & $75,035 \pm 1$ \\
\hline 35 & $75,043 \pm 1$ \\
\hline 36 & $75,049 \pm 1$ \\
\hline 37 & $75,055 \pm 1$ \\
\hline 38 & $75,060 \pm 1$ \\
\hline 39 & $75,065 \pm 1$ \\
\hline 40 & $75,070 \pm 1$ \\
\hline 41 & $75,074 \pm 1$ \\
\hline 42 & $75,078 \pm 1$ \\
\hline 43 & $75,082 \pm 1$ \\
\hline 44 & $75,085 \pm 1$ \\
\hline 45 & $75,088 \pm 1$ \\
\hline 46 & $75,091 \pm 1$ \\
\hline 47 & $75,094 \pm 1$ \\
\hline 48 & $75,096 \pm 1$ \\
\hline 49 & $75,099 \pm 1$ \\
\hline 50 & $75,101 \pm 1$ \\
\hline
\end{tabular}

reported in Table 6 for $6 \mathrm{p}^{4}\left({ }^{3} \mathrm{P}\right) n \mathrm{p}{ }^{2} \mathrm{P}_{3 / 2}$ levels between $n=8$ and $n=50$. Here also, as for the $6 \mathrm{p}^{4} n$ d series, the uncertainties affecting our calculated values were estimated with $\varepsilon\left(E_{\text {ion }}\right)=0.7 \mathrm{~cm}^{-1}$, $\varepsilon(R)=0.03 \mathrm{~cm}^{-1}$ and $\varepsilon\left(\delta_{\mathrm{nl}}\right)=0.05$.

Finally, it is interesting to note that, when arbitrarily doubling the uncertainty affecting the quantum defect from $\varepsilon\left(\delta_{n l}\right)=0.05$ to $\varepsilon\left(\delta_{n l}\right)=0.1$, the energy level uncertainties reported in Tables 5 and 6 were found to be typically increased by a factor of two for the lowest $n$-values and unchanged from $n \approx 30$ along the $6 \mathrm{p}^{4} n \mathrm{p}$ and $6 \mathrm{p}^{4} n \mathrm{~d}$ series.

\section{Conclusion}

Different physical models based on the pseudo-relativistic Hartree-Fock method have been used for modelling the atomic structure and for computing radiative parameters in neutral astatine, the rarest element on Earth. These calculations allowed us to provide for the first time a reliable spectroscopic designation to some energy levels experimentally observed but not clearly classified in the $6 p^{5}, 6 p^{4} 7 s, 6 p^{4} 7 p, 6 p^{4} 9 p$ and $6 p^{4} 10 p$ configurations. A semi-empirical approach based on the quantum defect formula has also been used for predicting level energies along the $6 \mathrm{p}^{4} \mathrm{np}$ and
Table 6

Predicted energies for the ${ }^{2} \mathrm{P}_{3 / 2}$ levels along the $6 \mathrm{p}^{4}\left({ }^{3} \mathrm{P}\right) \mathrm{np}$ Rydberg series from $\mathrm{n}=8$ to $\mathrm{n}=50$, as obtained using the quantum defect formula with $\mathrm{E}_{\text {ion }}=75,150.8 \pm 0.7 \mathrm{~cm}^{-1}$, $\mathrm{R}=109,737.03 \pm 0.03 \mathrm{~cm}^{-1}$ and $\delta_{\mathrm{nl}}=4.36 \pm 0.05$.

\begin{tabular}{|c|c|}
\hline$n$ & $\mathrm{E}\left(\mathrm{cm}^{-1}\right)$ \\
\hline 8 & $66,869 \pm 228$ \\
\hline 9 & $70,054 \pm 110$ \\
\hline 10 & $71,701 \pm 61$ \\
\hline 11 & $72,662 \pm 37$ \\
\hline 12 & $73,271 \pm 25$ \\
\hline 13 & $73,681 \pm 17$ \\
\hline 14 & $73,970 \pm 12$ \\
\hline 15 & $74,181 \pm 9$ \\
\hline 16 & $74,341 \pm 7$ \\
\hline 17 & $74,464 \pm 5$ \\
\hline 18 & $74,561 \pm 4$ \\
\hline 19 & $74,639 \pm 4$ \\
\hline 20 & $74,702 \pm 3$ \\
\hline 21 & $74,754 \pm 2$ \\
\hline 22 & $74,798 \pm 2$ \\
\hline 23 & $74,835 \pm 2$ \\
\hline 24 & $74,866 \pm 2$ \\
\hline 25 & $74,893 \pm 1$ \\
\hline 26 & $74,916 \pm 1$ \\
\hline 27 & $74,937 \pm 1$ \\
\hline 28 & $74,954 \pm 1$ \\
\hline 29 & $74,970 \pm 1$ \\
\hline 30 & $74,984 \pm 1$ \\
\hline 31 & $74,996 \pm 1$ \\
\hline 32 & $75,007 \pm 1$ \\
\hline 33 & $75,017 \pm 1$ \\
\hline 34 & $75,026 \pm 1$ \\
\hline 35 & $75,034 \pm 1$ \\
\hline 36 & $75,041 \pm 1$ \\
\hline 37 & $75,048 \pm 1$ \\
\hline 38 & $75,054 \pm 1$ \\
\hline 39 & $75,059 \pm 1$ \\
\hline 40 & $75,064 \pm 1$ \\
\hline 41 & $75,069 \pm 1$ \\
\hline 42 & $75,073 \pm 1$ \\
\hline 43 & $75,077 \pm 1$ \\
\hline 44 & $75,081 \pm 1$ \\
\hline 45 & $75,084 \pm 1$ \\
\hline 46 & $75,088 \pm 1$ \\
\hline 47 & $75,090 \pm 1$ \\
\hline 48 & $75,093 \pm 1$ \\
\hline 49 & $75,096 \pm 1$ \\
\hline 50 & $75,098 \pm 1$ \\
\hline
\end{tabular}

$6 \mathrm{p}^{4} n \mathrm{~d}(n=8-50)$ Rydberg series. The new theoretical investigation reported in the present paper is expected to provide a useful support to future laser-spectroscopy experiments at ISOLDE and to check other theoretical methods that will be used to model the atomic structure and radiative processes in At I.

\section{Acknowledgements}

This work was performed thanks to the support of the Belgian Research Initiative on eXotic nuclei for atomic, nuclear and astrophysics studies - BRIX (BELSPO IAP-VII Network No. P7/12). PQ is Research Director of the Belgian Fund for Scientific Research F.R.S.FNRS. Financial support from this organization is also greatly acknowledged.

\section{References}

[1] Holleman AF, Wiberg E. Inorganic chemistry. Academic Press; 2001.

[2] Corson DR, MacKenzie KR, Segre E. possible production of radioactive isotopes of element 85.. Phys Rev 1940;57:459.

[3] Fry C, Thoennessen M. Discovery of the astatine, radon, francium, and radium isotopes. At Data Nucl Data Tables 2013;99:497-519. 
[4] Goriely S, Samyn M, Pearson JM. Further explorations of Skyrme-Hartree-Fock-Bogoliubov mass formulas. VII. Simultaneous fits to masses and fission barriers.. Phys Rev C 2007;75:064312.

[5] Bloomer W, McLaughlin WH, Neirinckx RD, Adelstein SJ, Gordon PR, Ruth TJ, et al. Astatine-211 - tellurium radiocolloid cures experimental malignant ascites. Science 1981;212:340-1.

[6] Brown I. Astatine-211: its possible applications in cancer therapy. Int J Rad Appl Instrum A 1986;37:789-98.

[7] Zalutsky MR, Reardon DA, Akabani G, Coleman RE, Friedman AH, Friedman HS, et al. Clinical experience with alpha-particle emitting ${ }^{211}$ At: treatment of recurrent brain tumor patients with ${ }^{211}$ At-labeled chimeric antitenascin monoclonal antibody 81C6. J Nucl Med 2008;49:30-8.

[8] Vaidyanathan G, Zalutsky M. Applications of ${ }^{211}$ At and ${ }^{221}$ Ra in targeted alphaparticle radiotherapy. Curr Radiopharm 2010;4:283-94.

[9] Gustafsson AM, Bäck T, Elgqvist J, Jacobsson L, Hultborn R, Albertsson P, et al. Comparison of therapeutic efficacy and biodistribution of ${ }^{213} \mathrm{Bi}$ - and 211 At-labeled monoclonal antibody MX35 in an ovarian cancer model. Nucl Med Biol 2012;39:15-22.

[10] Guérard F, Lee Y-S, Baidoo K, Gestin Jf, Brechbiel MW. Unexpected behaviour of the heaviest halogen astatine in the nucleophilic substitution of aryliodonium salts. Chem - A Euro J 2016;22:12332-9.

[11] McLaughlin R. Absorption spectum of astatine. J Opt Soc Am 1964;54:965-7.

[12] Rothe S, Andreyev AN, Antalic S, Borschevsky A, Capponi L, Cocolios TE, et al. Measurement of the first ionization potential of astatine by laser ionization spectroscopy. Nature Commun 2013;4:1835-40.

[13] Raeder S, Lassen J, Heggen H, Teigelhöfer A. In-source spectroscopy on astatine and radium for resonant laser ionization. Hyperfine Interact 2014;227:77-83.

[14] Palmeri P, Quinet P, Biémont E, Yushchenko AV, Jorissen A, Van Eck S. Radiative decay of the $4 \mathrm{~d}^{5}\left({ }^{6} \mathrm{~S}\right) 5 \mathrm{p} \mathrm{z}^{5,7} \mathrm{P}^{\circ}$ states in Tc II: comparsion along the homologous and isoelectronic sequences. Application to astrophysics. Mon Not R Astron Soc 2007;374:63-71.
[15] Fivet V, Quinet P, Biémont E, Jorissen A, Yushchenko AV, Van Eck S. Transition probabilities in singly ionized promethium and the identification of Pm II lines in Przybylski's star and HR 465. Mon Not R Astron Soc 2007;380:771-80.

[16] Quinet P. Investigation of intravalence, core-valence and core-core electron correlation effects in polonium atomic structure calculations. J Quant Spectr Rad Trans 2014;145:153-9.

[17] Biémont E, Quinet P, Van Renterghem V. Theoretical investigation of neutral francium. J Phys B: At Mol Opt Phys 1998;31:5301-14.

[18] Quinet P, Argante C, Fivet V, Terranova C, Yushchenko AV, Biémont E. Atomic data for radioactive elements Ra I, Ra II, Ac I and Ac II and application to their detection in HD 101065 and HR 465. Astron Astrophys 2007;474:307-14.

[19] Fivet V, Quinet P, Biémont E. Transition probabilities in complex ions: the case of americium. J Elec Spectrosc Rel Phen 2007;156-158:255-8.

[20] Cowan RD. The theory of atomic structure and spectra. Berkeley: University of California Press; 1981.

[21] Quinet P, Palmeri P, Biémont E, McCurdy MM, Rieger G, Pinnington EH, et al. Experimental and theoretical lifetimes, branching fractions and oscillator strengths in Lu II. Mon Not R Astron Soc 1999;307:934-40.

[22] Quinet P, Palmeri P, Biémont E, Li ZS, Zhang ZG, Svanberg S. Radiative lifetime measurements and transition probability calculations in lanthanide ions. J Alloys Comp 2002;344:255-9.

[23] Biémont E, Quinet P. Recent advances in study of lanthanide atoms and ions. Phys Scr T 2003;105:38-54.

[24] Fraga S, Karwowski J, Saxena KMS. Handbook of atomic Data. Amsterdam: Elsevier; 1976

[25] Luc-Koenig E, Morillon C, Vergès J. Experimental and theoretical studies in atomic iodine: infrared arc spectrum observations, classification and hyperfine structure. Phys Scr 1975;12:199-219. 